\title{
Computational study of the Dehydrogenation Effect of Dopant Ti on Nanostructured NaAlH4 Cluster
}

\author{
Xiaogang Tong ${ }^{1}$ and Hongshan Chen $^{1}$ \\ ${ }^{1}$ Northwest Normal University
}

September 28, 2020

\begin{abstract}
Employing the evolutionary algorithm combined with density functional theory and perturbation theory model, we investigate the geometric and electronic structures of pure and Titanium-doped Na4Al4H16 cluster to demonstrate the effect of dopant Ti on the dehydrogenation properties of the nanostructured NaAlH4. The result shows the Ti-doped Na4Al4H16 clusters are more stable thermodynamically, but the average dehydrogenation enthalpies and the energy barriers for H2 desorption are significantly decreased. Doping of $\mathrm{Ti}$ weakens the $\mathrm{Al}-\mathrm{H}$ bond, reduces the dehydrogenation temperatures, and the dehydrogenation performance of the NaAlH4 nanocluster is conspicuously promoted.
\end{abstract}

\section{Hosted file}

manuscript-submission.pdf available at https://authorea.com/users/362299/articles/483514computational-study-of-the-dehydrogenation-effect-of-dopant-ti-on-nanostructured-naalh4cluster 